\title{
Interstellar Scintillation as a Cosmological Probe: Prospects and Challenges $\dagger$
}

\author{
J. Y. Koay ${ }^{1 *}$, J.-P. Macquart ${ }^{1}$, B. J. Rickett ${ }^{2}$, H. E. Bignall ${ }^{1}$, \\ D. L. Jauncey ${ }^{3}$, J. E. J. Lovell ${ }^{4}$, C. Reynolds ${ }^{1}$, T. Pursimo ${ }^{5}$ \\ L. Kedziora-Chudczer ${ }^{6}$ and R. Ojha ${ }^{7}$ \\ ${ }^{1}$ International Centre for Radio Astronomy Research, Curtin University, Australia \\ *email: kevin.koay@icrar.org \\ ${ }^{2}$ Department of Electrical \& Computer Engineering, University of California, San Diego, USA \\ ${ }^{3}$ CSIRO Astronomy \& Space Science, Australia Telescope National Facility, Australia \\ ${ }^{4}$ School of Mathematics \& Physics, University of Tasmania, Australia \\ ${ }^{5}$ Nordic Optical Telescope, E-38700 Santa Cruz de La Palma, Spain \\ ${ }^{6}$ School of Physics \& Astrophysics, University of New South Wales, Australia \\ ${ }^{7}$ Institute for Astrophysics \& Computational Sciences, Catholic University of America, USA
}

\begin{abstract}
The discovery that interstellar scintillation (ISS) is suppressed for compact radio sources at $z \gtrsim 2$ has enabled ISS surveys to be used as cosmological probes. We discuss briefly the potential and challenges involved in such an undertaking, based on a dual-frequency survey of ISS carried out to determine the origin of this redshift dependence.
\end{abstract}

Keywords. intergalactic medium, galaxies: active, radio continuum: ISM, scattering

\section{Prospects}

The radio variability observed in many compact active galactic nuclei (AGN) at intrahour and intra-day time-scales has been linked to scintillation caused by scattering in the interstellar medium (ISM) of our own Galaxy (Kedziora-Chudczer et al. 1997; Jauncey \& Macquart 2001; Rickett et al. 2001; Dennett-Thorpe \& de Bruyn 2002). Two factors make interstellar scintillation (ISS) an ideal probe of the physics of the ISM and of the background sources themselves. (1) The amplitude of scintillation decreases with increasing source angular size (thus, in the case of atmospheric scintillation at optical wavelengths, stars twinkle but planets do not), so ISS can be utilized to probe source sizes down to $\mu$ as scales (Lazio et al. 2004), which is orders of magnitude better than any ground-based radio telescope can achieve. (2) The phenomenon is pervasive; the $5 \mathrm{GHz}$ Microarcsecond Scintillation Induced Variability (MASIV) Survey found that $\sim 60 \%$ of flat-spectrum AGNs exhibit 2-10\% rms flux density variations due to scintillation (Lovell et al. 2008).

Both the fraction of scintillating sources and their scintillation amplitudes have been found to decrease for sources with redshifts $z \gtrsim 2$ (Lovell et al. 2008), thereby imbuing ISS surveys with a cosmological significance. What causes that redshift dependence of ISS? Possible explanations include (1) a $(1+z)^{0.5}$ scaling of angular size due to cosmological expansion (Rickett et al. 2007) in a flux-limited and brightness temperature-limited sample of sources, (2) selection effects due to the redshift dependence of source luminosities, Doppler boosting factors or spectral indices, (3) evolution of AGN morphology, (4)

$\dagger$ Presented on behalf of J.Y. Koay by J.-P. Macquart. 
scatter broadening in the ionized intergalactic medium (IGM) due to multi-path propagation (Rickett et al. 2007), and (5) weak gravitational lensing by foreground objects.

There are great prospects for ISS as a cosmological probe at $\mu$ as resolution. Investigations into its redshift dependence can potentially probe the angular size-redshift relation of flat-spectrum AGNs, AGN evolution, turbulence in the IGM, and/or the distribution of matter in the Universe; at the very least it will place strong constraints on them.

\section{Observations and Challenges}

We observed 140 compact, flat-spectrum AGNs at $0 \lesssim z \lesssim 4$ to investigate the origin of the redshift dependence of ISS. The sources were observed simultaneously at $4.9 \mathrm{GHz}$ and 8.4 GHz on two VLA sub-arrays; each was observed for one minute at 2-hour intervals for a total duration of 11 days, scheduled in sidereal time. The main motivation for the observations was to determine whether the redshift dependence of ISS scales with frequency, since scatter broadening, intrinsic source size effects and space-time curvature each scale differently with frequency. The observations also function as a precursor for future larger-scale surveys with next-generation radio telescopes, providing a platform for exploring the challenges (discussed below) involved in such an experiment.

- Instrumental and Systematic Errors. Possible errors in the time domain are fluxdependent errors (variable calibrators, residual gain and pointing errors) and fluxindependent errors (system noise, resolved sources, and confusion). Many of them appear as flux-density variations that repeat daily, since our observations were scheduled in sidereal time. We developed various methods to estimate them-see Koay et al. (2011).

- Intrinsic Source Variability. Owing to their compact nature, some of the sources may exhibit intrinsic variations that are difficult to deconvolve from ISS. The variability amplitudes of our sources were found to be significantly correlated with line-of-sight Galactic $\mathrm{H} \alpha$ intensities (Koay et al. 2011), confirming that ISS dominates their variability on a statistical level at least.

- Source Selection Biases. Since our survey was flux-limited and involved relativistically beamed sources, it is likely that the high-redshift sources are more luminous and have larger Doppler boosting factors. The fact that the sources are observed at increasing rest-frame emission frequencies with increasing redshift adds to the bias. We devised a means of mitigating those effects by examining the ratio of the variability amplitudes at the two frequencies for each source individually, and then comparing the distribution of the ratios at high and low redshift.

- Complexity of ISM and Source Properties. The anisotropy and inhomogeneity of the ISM, coupled with the complexity of source structures, complicate the interpretation of the redshift dependence. We used Monte-Carlo simulations to model the statistics of ISS amplitudes for comparisons with observed distributions.

\section{References}

Dennett-Thorpe, J. \& de Bruyn, A. G. 2002, Nature, 415, 57

Jauncey, D. L. \& Macquart, J.-P. 2001, A\&A, 370, L9

Kedziora-Chudczer, L. et al. 1997, ApJ, 490, L9

Kedziora-Chudczer, L. et al. 2001, MNRAS, 325, 1411

Koay, J. Y., et al. 2011, AJ, 142, 108

Lazio, T. J. W., et al. 2004, New Astron. Revs, 48, 1439

Lovell, J. E. J., et al. 2008, ApJ, 689, 108

Rickett, B. J., et al. 2001, ApJ, 550, L11

Rickett, B., et al. 2007, Proc. Science, 46, PoS(MRU)046 